\title{
ESTUDIOS SOBRE EL COMPORTAMIENTO DE FORRAJEO DE Acromyrmex lundi Guering (HYMENOPTERA, FORMICIDAE) Y SU EFECTO SOBRE EL CRECIMIENTO DE PROCEDENCIAS DE Eucalyptus globulus Labill. (Myrtaceae) $^{1}$
}

\author{
Carmen Lidia Martínez ${ }^{2}$, María Begoña Riquelme Virgala ${ }^{3}$, Marina Vilma Santadino ${ }^{3}$, Ana María de Haro ${ }^{3}$ \\ e Justo José Barañao ${ }^{3}$
}

\begin{abstract}
RESUMEN - El ataque de las hormigas cortadoras es una de las principales razones de pérdida de plantas durante la etapa de establecimiento de un monte forestal. Los objetivos de este estudio fueron evaluar la intensidad de forrajeo de Acromyrmex lundi asociado a algunas condiciones ambientales y procedencias de Eucalyptus globulus y estimar el impacto de la herbivoría sobre el crecimiento inicial de estos árboles. Dos ensayos se llevaron a cabo durante dos temporadas estivo-otoñales consecutivas, en el Campo Experimental de la Universidad Nacional de Luján, Argentina. Los materiales de diferentes procedencias fueron dispuestos en bloques equidistantes a $5 \mathrm{~m}$ de un nido activo de A. lundi. Durante doce semanas se contabilizó el número de hojas cortadas por las hormigas y se registraron algunas variables meteorológicas. En el segundo año la mitad de las parcelas fue protegida de las hormigas y se midieron variables de crecimiento. Los materiales de todas las procedencias fueron atacadas por A. lundi, aunque el número de hojas cosechada fue significativamente diferente sólo entre los orígenes Flinders Island (52,5 hojas) y Nullo Mountain (28,5 hojas). El número de días lluviosos fue el único factor ambiental que se relacionó positivamente con la actividad de las hormigas. Todos los tratamientos redujeron significativamente la altura, el diámetro y el área foliar cuando fueron atacados por las hormigas. Las distintas procedencias compensaron diferencialemnte la herbivoría. Se discuten los resultados en función de los criterios a tener en cuenta para la selección de materiales genéticos como herramienta de manejo y control del ataque de $A$. lundi.
\end{abstract}

Palabras-clave: Eucalipto; Hormigas cortadoras; Vivero forestal.

\section{ESTUDO DO COMPORTAMENTO DE FORRAGEAMENTO DE AcromyrmeX lundi Guering (HYMENOPTERA, FORMICIDAE) E SEU EFEITO SOBRE O CRESCIMENTO DE PROCEDÊNCIAS DE Eucalyptus globulus Labill. (Myrtaceae)}

\begin{abstract}
RESUMO - O ataque de formigas-cortadeiras é uma das principais razões da perda de plantas durante a fase de implementação de um plantio florestal. Os objetivos deste estudo foram avaliar a intensidade do ataque de forrageamento Acromyrmex lundi associada a algumas condições ambientais e procedências de Eucalyptus globulus e estimar o impacto do ataque dos insetos (herbivoria) sobre o crescimento inicial dessas árvores. Dois ensaios foram realizados por duas temporadas consecutvas (verão-outono) na área Experimental da Universidade Nacional de Luján, na Argentina. O material de diferentes procedências foi arranjado em blocos equidistantes a $5 \mathrm{~m}$ de um ninho ativo de A. Iundi. Durante 12 semanas, contabilizou-se o número
\end{abstract}

\footnotetext{
${ }^{1}$ Recebido em 25.02.2013 aceito para publicação em 19.11.2014

${ }^{2}$ In memorian.

${ }^{3}$ Universidad Nacional de Luján, Departamento de Tecnología, Luján, Buenos Aires, Argentina. E-mail:<mriquelme@agro.uba.ar>, <msantadino@hotmail.com>e<justobaranao@yahoo.com.ar>.
} 
de folhas cortadas por formigas, e algumas variáveis meteorológicas foram registradas. No segundo ano, a metade das parcelas foi protegida das formigas, e as variáveis de crescimento foram mensuradas. O material de todas as procedências foi atacado por A. lundi, embora o número de folhas colhidas foi diferente significativamente apenas entre as origens Flinders Island (52,5 folhas) e Nullo Mountain Nullo (28,5 folhas). O número de dias de chuva foi o único fator ambiental positivamente relacionado com a atividade das formigas. Todos os tratamentos reduziram significativamente a altura, o diâmetro e a área foliar quando atacados por formigas. Os resultados são discutidos em função dos critérios a serem considerados na seleção de material genético como ferramenta para o manejo e combate ao ataque de A. Iundi.

Palavras-chave: Eucalipto; Formigas-cortadeiras; Viveiro florestal.

\section{INTRODUCCIÓN}

Según el último inventario nacional forestal, de las 18 a 20 millones de hectáreas de suelos con aptitud forestal que posee la Argentina, cerca de 200 mil hectáreas se utilizan para el cultivo de eucalipto, ocupando el segundo lugar en superficie implantada luego de las coníferas, con la provincia de Buenos Aires como una de las más importantes en superficie y producción luego de las provincias mesopotámicas (SAGPyA, 2001). Las principales especies utilizadas en el país son Eucalyptus camaldulensis Dehnh., E. tereticornis Smith., E. globulus Labill., E. dunii Maiden., E. viminalis Labill., E. saligna Smith. y E. grandis Hill., con destino a la industria de papel y de madera sólida (VILLEGAS; RIVERA, 2002). E. globulus conocido en inglés como bluegum eucalyptus, es la especie más cultivada en el mundo y de ella se reconocen cuatro subespecies: globulus, maidenii, bicostata y pseudoglobulus, todas ellas originarias de Tasmania y Australia (SKOLMEN; LEDING, 1990).

Uno de los principales factores que causan la pérdida de plantas de eucalipto en el período de implantación es el ataque de hormigas cortadoras. Estos insectos, de origen Neotropical, pertenecen a los géneros Atta Fabricius, 1805 y Acromyrmex Mayr, 1865, incluidos en la tribu Attini. El efecto de la cosecha de hojas puede provocar la muerte de las plantas o su menor y/o más lento crecimiento y por lo tanto afectar la producción final de madera (ZANETTI et al., 2000; DELLA LUCIA, 2003).

Una de las formas de combatir una plaga es a través de la selección de materiales genéticos menos preferidos o más tolerantes a su ataque. Aunque las hormigas cortadoras pueden utilizar una amplia variedad de plantas, su preferencia de corte depende de varios factores, como la calidad de los nutrientes presentes en las hojas, su edad, su dureza y la presencia de determinados metabolitos secundarios (FOLGARAIT et al., 1994;
PELOTTO; DEL PERO DEMARTÍNEZ, 2002; DELABIE et al., 2003; SOUZA-SILVA; ZANETTI, 2007), así como las necesidades estacionales y el tamaño de la colonia (FARJI BRENER, 1993; PILATI et al., 1997; ALVES; DELLA LUCIA, 1999). El forrajeo selectivo de las Attini ha sido observado en relación a diversas plantas (ROCKWOOD, 1976; HOWARD, 1987; NICHOLSORIANS, 1991a,b; PILATI et al., 1997; RODRIGUEZ et al., 2008) y en particular entre diferentes especies de eucaliptos (SANTANA; COUTO, 1990; CAFFARINI et al., 2006; MARSARO JÚNIOR et al., 2007). El follaje de los eucaliptos posee una alta concentración de metabolitos secundarios tales como taninos, otros fenoles y aceites esenciales que podrían tener una influencia significativa en la interacción de estos insectos con las especies de este género (OHMART y EDWARDS, 1991).

Algunos factores ambientales también han sido estudiados en relación a su efecto sobre la intensidad, el patrón y la velocidad de forrajeo de las hormigas cortadoras, encontrándose diversos resultados según el lugar, la especie vegetal y la especie de hormiga (GOMIDES et al., 1997; DIMARCO et al., 2004; CAFFARINI et al., 2006; SÁNCHEZ GALARZA; URCUQUI BUSTAMANTE, 2006).

Este estudio tuvo como objetivos evaluar la intensidad de cosecha de $A$. lundi asociada a algunas condiciones ambientales y procedencias de Eucalyptus globulus y determinar el efecto de la herbivoría sobre el crecimiento inicial de estos árboles en condiciones de campo.

\section{MATERIALES Y MÉTODOS}

\subsection{Condiciones de estudio}

Los estudios se llevaron a cabo en el Campo experimental de la Universidad Nacional de Luján(UNLu), provincia de Buenos Aires, Argentina durante dos etapas 
estivo-otoñales consecutivas. La ciudad de Luján está situada a los $34^{\circ} 35^{\prime}$ de latitud Sur y $59^{\circ} 05^{\prime}$ de longitud Occidental, tiene clima templado con temperatura media de $10{ }^{\circ} \mathrm{C}$ en invierno y $23{ }^{\circ} \mathrm{C}$ en verano, con heladas en otoño, invierno y primavera. La temperatura máxima, la temperatura mínima y las precipitaciones fueron registradas diariamente durante ambos ensayos por la Estación meteorológica de la UNLu.

Se utilizaron plantas de ocho meses correspondientes a once procedencias de cuatro subespecies de Eucalyptus globulus (Tabla 1) plantadas en macetas de polietileno de cinco litros de capacidad. Cada hoja fue numerada con tinta indeleble en su cara adaxial en orden correlativo desde la base de la planta y en cada fecha de observación se continuó la enumeración en las hojas nuevas. Las plantas fueron desbrotadas, reduciéndolas al eje central, durante todo el estudio y recibieron riego suplemetario.

Las macetas se ubicaron dentro de trincheras (bloques), dispuestas en semicírculo y con orientación SE-NO, a cinco metros de un hormiguero activo de A. lundi. La cubierta herbácea que rodeó el ensayo se mantuvo cortada al ras en un radio de siete metros para restringir al máximo la oferta extra de sustrato para cosechar.

El primer ensayo siguió un diseño experimental en bloques con siete repeticiones. Cada bloque contó con cuatro plantas de cada origen dispuestas juntas en orden aleatorio dentro del bloque. En el segundo año el diseño fue en bloques divididos con cuatro réplicas, donde la mitad de cada bloque fue protegido del ataque de las hormigas por medio de la aplicación de insecticidas en su perímetro. El bloque principal fueron los orígenes y los subbloques la herbivoría. Cada subbloque tuvo tres plantas por origen asignadas al azar.

\subsection{Evaluación de la preferencia por procedencias}

Para evaluar la preferencia de $A$. lundi por las procedencias de E. globulus, se efectuaron observaciones del número de hojas cortadas por las hormigas cada seis a diez días durante doce semanas. El período de recuento abarcó desde febrero hasta mayo en el primer ensayo y desde de enero hasta abril en el segundo. Se consideró una hoja cortada a aquella que presentó más del $50 \%$ del folíolo cortado por las hormigas. No se observó ningún otro agente animal que pudiera provocar un síntoma similar.
Tabla 1 - Subespecies y procedencias de Eucalyptus globulus usados en el ensayo.

Tabela 1 - Subespécies e procedências de Eucalyptus globulus utilizados no ensaio.

\begin{tabular}{ll}
\hline \multicolumn{1}{c}{ Subspecie } & \multicolumn{1}{c}{ Procedencia } \\
\hline E. globulus globulus & Otway National Park (Otw) \\
& Flinders Island (Fli) \\
& Jeeralang (Jee) \\
& Moogara (Moo) \\
& Murrabrine (Mur) \\
E. globulus maidenii & Tantawango (Tan) \\
& Wog Way (Wog) \\
& Bolaro Mountain (Bol) \\
& Wee Jasper (Wee) \\
E. globulus bicostata & Nullo Mountain (Nul) \\
& Wiebens (Wie) \\
\hline
\end{tabular}

La variable estudiada fue el número total de hojas cortadas de cada origen. Los datos fueron sometidos a un análisis de varianza con un tratamiento (orígenes), previa transformación a Raiz $(\mathrm{x}+0,5)$ para normalizarlos $y$ cuando el ANOVA resultó significativo $(p<0,05)$, las medias fueron comparadas con la prueba de Tuckey.

\subsection{Variables climáticas}

Para analizar el efecto de algunas condiciones ambientales sobre la actividad de forrajeo se realizó un análisis de regresión múltiple utilizando los datos de ambos períodos. La variable dependiente fue el número de hojas cortadas de todas las procedencias, mientras que las variables regresoras analizadas fueron la temperatura máxima, la temperatura mínima, la precipitación media diaria y el número de días con precipitaciones. Las variables cuyo coeficiente de regresión fue no significativo ( $\mathrm{p}>0,1)$, fueron eliminadas del modelo(BARÓN LÓPEZ; TÉLLEZ MONTIEL, 2004).

\subsection{Evaluación del crecimiento}

Para evaluar el efecto de la cosecha de hojas sobre el crecimiento de las plantas de eucalipto, durante el segundo ensayo se registraron en todas las plantas la altura inicial $\left(\mathrm{H}_{\mathrm{i}}\right)$ y final $\left(\mathrm{H}_{\mathrm{f}}\right)$ desde el cuello hasta el ápice, con las que se calculó el crecimiento: $\mathrm{C}=$ $\mathrm{H}_{\mathrm{f}}-\mathrm{H}_{\mathrm{i}}$; el área foliar inicial $\left(\mathrm{AF}_{\mathrm{i}}\right)$ y final $\left(\mathrm{AF}_{\mathrm{f}}\right)$ y el diámetro final del tallo a nivel del cuello $\left(\mathrm{D}_{\mathrm{f}}\right)$. Para el cálculo del área foliar se midieron el largo (L) y ancho máximo (A) de todas las hojas y se utilizaron las fórmulas propuestas por Guarnaschelli (2009) para cada procedencia.

Revista Árvore, Viçosa-MG, v.39, n.1, p.189-198, 2015 
Se compararon el crecimiento de las plantas, el incremento del área foliar y el diámetro final con y sin ataque de hormigas a través de un análisis de varianza para un diseño en bloques divididos, con dos tratamientos (Herbivoría y Procedencias) (STEEL; TORRIE, 1980).

Se utilizó el programa Statistica (STATSOFT, 2000).

\section{RESULTADOS}

\subsection{Preferencia por orígenes}

En la Figura 1 se puede observar el número de hojas por planta cortadas durante todo el período de observación en los dos ensayos. En ambos años, todas las subespecies y procedencias fueron cosechadas por las hormigas, aunque sólo en el segundo estudio se observaron diferencias significativas en la herbivoría entre los materiales genéticos $\left(\mathrm{F}_{10,30}=3,24 ; \mathrm{p}=0,006\right)$. E. globulus bicostata Nullo Mountain fue la procedencia que presentó el menor número de hojas cortadas al final del estudio $(28,5 \pm 5,07)$, significativamente menos que la más atacada, E. globulus globulus Flinders Island, con 52,5 \pm 11.01 hojas cosechadas.

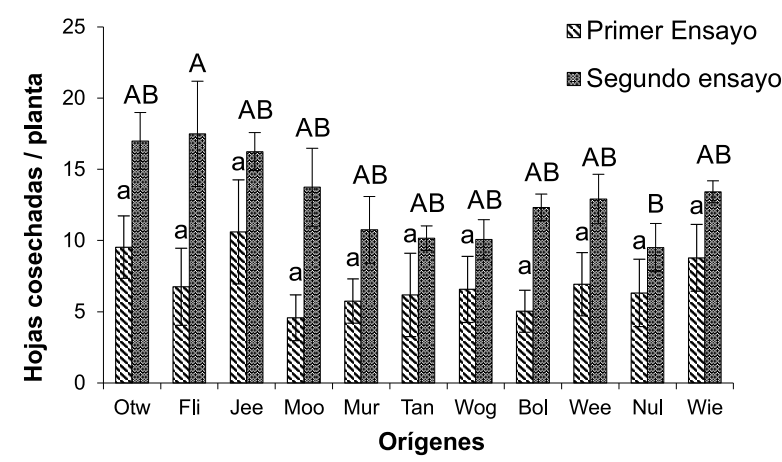

Figura 1 - Número de hojas por planta (media \pm EE) de diferentes procedencias de Eucalyptus globulus cosechadas por Acromyrmex lundi durante dos temporadas estivo-otoñales. a-Primer ensayo $n=7$ Segundo ensayo $n=4$. Letras diferentes en un mismo ensayo indican que hay diferencias significativas entre tratamientos (Tuckey, $\mathrm{p}<0.05$ ).

Figura 1 - Número de folhas por planta (média \pm EP) de diferentes procedências de Eucalyptus globulus cortados por Acromyrmex lundi por duas temporadas (verão-outono). a- primeiro ensaio $(n=4)$; e $b$ - segundo ensaio $(n=7)$. Letras diferentes no mesmo teste indicam que existem diferenças significativas entre os tratamentos (Tuckey, $p<0.05$ ).

\subsection{Efecto de las variables climáticas}

El único factor climático que se relacionó con la intensidad de forrajeo fue el número de días con lluvia a través de un modelo lineal positivo $\left(\mathrm{R}^{2}=0,37 ; \mathrm{F}=\right.$ 9,99; $\mathrm{p}=0,006)$ :

$\mathrm{y}$ (número de hojas cosechadas) $=97,95+48,18$ x (número de días con lluvia)

El signo positivo de la pendiente del modelo indica que la actividad de las hormigas aumenta cuando hay más días con lluvia dentro del rango registrado en los estudios ( 0 a7 días con lluvias entre 0,1 y $87,0 \mathrm{~mm}$ por día).

\subsection{Efecto de la herbivoría sobre el crecimiento}

El efecto de la herbivoría sobre las variables de crecimiento puede observarse en la Tabla 2. Se encontraron diferencias significativas entre los árboles con y sin herbivoría en el crecimiento en altura $\left(\mathrm{F}_{1,2}=247,30\right.$; $\mathrm{p}=0,004)$, en el aumento del área foliar $\left(\mathrm{F}_{1,2}=29,35\right.$; $\mathrm{p}=0,032)$ y en el diámetro final $\left(\mathrm{F}_{1,2}=31,75 ; \mathrm{p}=0,030\right)$, presentándose todas las variables reducidas en las plantas que sufrieron herbivoría, independientemente de la procedencia. La excepción fue la procedencia Otways Island cuyo diámetro medio fue levemente mayor en las plantas con defoliación (Tabla 2). Entre las demás procedencias, Bolaro de la subespecie E. globulus maidenii fue la más afectada por las hormigas, presentando una reducción de $65,2 \%$ en el crecimiento en altura, 34,8\% en el diámetro final y $67,0 \%$ en el aumento del área foliar. En el otro extremo, el material Tantawango de la subespecie E. globulus maidenii fue el menos afectado en su crecimiento en altura $(8,6 \%)$ y E. globulus bicostata Nullo Mountain fue la procedencia que sufrió la menor reducción del diámetro $(1,5 \%)$ y del incremento de área foliar (9,3\%), de acuerdo con la Figura 2.

\section{DISCUSIÓN}

Acromyrmex lundi cosechó hojas de todas las procedencias de Eucalyptus globulus evaluadas en este estudio sin presentar una marcada selectividad entre las mismas. Un estudio sobre la actividad de Acromyrmex lobicornis Emery en el norte de la Patagonia concluyó que el forrajeo de las hormigas cortadoras de hojas está relacionado a la calidad nutricional de los recursos disponibles y a la abundancia relativa de los mismos, actuando bajo una combinación de comportamientos selectivos y oportunistas según la estación del año y la colonia (FRANZEL; FARJ BRENER,

Revista Árvore, Viçosa-MG, v.39, n.1, p.189-198, 2015

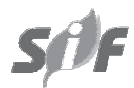


Tabla 2 - Crecimiento en altura $(\mathrm{cm})$, aumento del area foliar $\left(\mathrm{cm}^{2}\right)$ y diámetro del tallo $(\mathrm{cm})$ (media-error estándar) de plantas de Eucalyptus globulus de distintas procedencias expuestas $(\mathrm{C} / \mathrm{H})$ y no expuestas $(\mathrm{S} / \mathrm{H})$ durante 12 semanas a la herbivoría por Acromyrmex lundi.

Tabela 2 - Crescimento em altura $(\mathrm{cm})$, aumento da área foliar $\left(\mathrm{cm}^{2}\right)$ e diâmetro $(\mathrm{cm})$ (média erro-padrão) de plantas de Eucalyptus globulus de várias procedências, expostas (C/H) e não expostas (S/H), durante 12 semanas, a herbivoria por Acromyrmex lundi.

\begin{tabular}{|c|c|c|c|c|c|c|}
\hline \multirow{2}{*}{ Origen $(*)$} & \multicolumn{2}{|c|}{ Crecimiento } & \multicolumn{2}{|c|}{ Area foliar } & \multicolumn{2}{|c|}{ Diámetro } \\
\hline & $\mathrm{C} / \mathrm{H}$ & $\mathrm{S} / \mathrm{H}$ & $\mathrm{C} / \mathrm{H}$ & $\mathrm{S} / \mathrm{H}$ & $\mathrm{C} / \mathrm{H}$ & $\mathrm{S} / \mathrm{H}$ \\
\hline Otw & $55,47(6,24)$ & $63,04(8,51)$ & $552,05(168,13)$ & $900,76(316,64)$ & $1,15(0,15)$ & $1,11(0,06)$ \\
\hline Fli & $52,73(4,34)$ & $64,00(3,62)$ & $459,80(91,19)$ & $729,29(167,05)$ & $1,01(0,05)$ & $1,04(0,03)$ \\
\hline Jee & $48,76(8,89)$ & $58,11(3,04)$ & $994,95(226,17)$ & $1249,79(282,31)$ & $1,03(0,02)$ & $1,10(0,09)$ \\
\hline Moo & $57,69(7,59)$ & $74,63(4,12)$ & $469,22(142,57)$ & $961,39(61,30)$ & $0,94(0,04)$ & $1,09(0,05)$ \\
\hline Mur & $45,36(1,98)$ & $63,00(7,86)$ & $392,69(1,63)$ & $976,27(166,62)$ & $0,93(0,10)$ & $1,12(0,02)$ \\
\hline Tan & $64,00(2,43)$ & $70,00(5,26)$ & $864,55(86,45)$ & $1039,27(195,10)$ & $0,96(0,03)$ & $0,99(0,02)$ \\
\hline Wog & $52,67(6,58)$ & $74,84(4,16)$ & $1014,01(111,92)$ & $1405,64(239,53)$ & $0,97(0,03)$ & $1,04(0,02)$ \\
\hline Bol & $22,61(12,40)$ & $65,00(0,60)$ & $332,31(170,07)$ & $1007,32(81,85)$ & $0,64(0,06)$ & $0,99(0,02)$ \\
\hline Wee & $44,04(6,61)$ & $70,83(7,44)$ & $750,65(153,07)$ & $1388,09(190,92)$ & $0,92(0,04)$ & $1,11(0,04)$ \\
\hline Nul & $45,60(10,45)$ & $62,52(7,38)$ & $1619,68(420,13)$ & $1786,76(299,86)$ & $1,07(0,19)$ & $1,08(0,01)$ \\
\hline Wie & $59,44(12,66)$ & $80,68(6,67)$ & $808,78(356,50)$ & $1154,12(176,03)$ & $1,06(0,09)$ & $1,19(0,02)$ \\
\hline Media $(* *)$ & $49,85 \mathrm{a}$ & $67,88 \mathrm{~b}$ & $750,85 \mathrm{a}$ & $1145,34 \mathrm{~b}$ & $0,97 \mathrm{a}$ & $1,08 \mathrm{~b}$ \\
\hline
\end{tabular}

(*) Otw (Otway); Fli (Flinders); Jee (Jeeralang); Moo (Moogara); Mur (Murrabrine); Tan (Tantawanglo); Wog (Wog Way); Bol (Bolaro); Wee (Weesjasper); Nul (Nullo); Wie (Wiebens).

$(* *)$ Letras distintas correspondientes a las medias de una misma variable presentan diferencias significativas. ANOVA $p<0,05$.

(**) Letras diferentes correspondentes à média da mesma variável diferem significativamente. ANOVA $\mathrm{p}<0,05$.

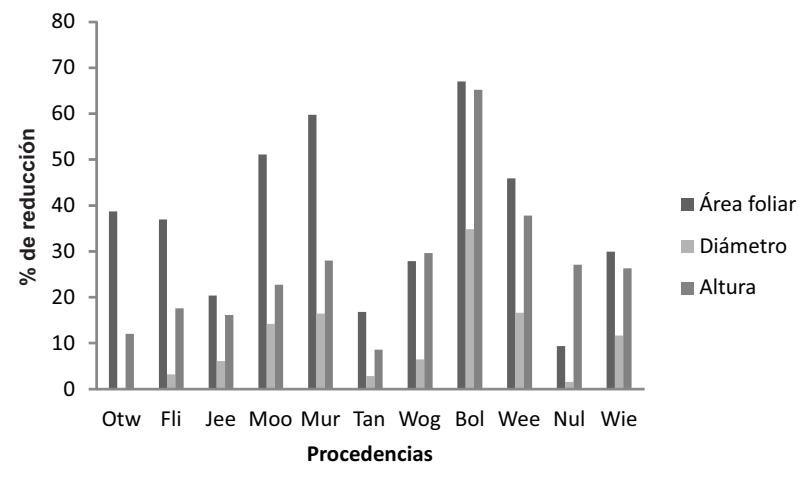

Figura 2 - Porcentaje medio de reducción de la altura, el diámetro y el área foliar de plantas de distintas procedencias de Eucalyptus globulus expuestas a herbivoría por Acromyrmex lundi.

Figura 2 - Redução porcentual média de altura, diâmetro e área foliar das plantas de diferentes procedências de Eucalyptus globulus expostos à herbivoria por Acromyrmex lundi.

2000). En el presente trabajo, todas las procedencias fueron ofrecidas en igual cantidad de plantas, por lo que el factor disponibilidad del recurso no pudo influir en el forrajeo, determinando que la respuesta de las hormigas estuviera regida principalmente por la calidad del mismo. En el segundo ensayo, este factor pudo haber sido el determinante en el forrajeo selectivo, registrándose casi el doble de hojas cortadas en $E$. globulus globulus Flinders Island con respecto a la procedencia Nullo Mountain de la subespecie E. globulus bicostata.Algunos autores han registrado para otras hormigas cortadoras preferencia entre especies y entre procedencias de una misma especie de Eucalyptus (SANTANA; COUTO, 1990; MARSARO JÚNIOR et al., 2007) e incluso se ha observado una actividad de forrajeo preferencial entre diferentes tipos de hojas de una misma especie de eucalipto, comportamiento asociado a la distinta concentración de compuestos secundarios (ALVES; DELLALUCIA, 1999). En particular para A. lundi, Caffarini et al. (2006) estudiaron el efecto del estrés hídrico sobre el área foliar removida de diferentes procedencias de E. globulus, encontrando que E. globulus bicostata Nullo Mountain fue una de las que sufrió el menor porcentaje de herbivoría, aunque no estadísticamente diferente del resto de las procedencias, entre las que se encontraban la mayoría de las estudiadas en este trabajo aunque no Flinders Island. Sin embargo, estos mismos autores en laboratorio evaluaron el tiempo de acarreo y en este caso Nullo Mountain fue una de las procedencias que más rápidamente se llevaron las hormigas al nido.

Revista Árvore, Viçosa-MG, v.39, n.1, p.189-198, 2015 
En relación a las variables climáticas estudiadas, sólo el número de días con precipitaciones se relacionó con la intensidad de cosecha. Farji Brener (1993) estudió la influencia de la estacionalidad sobre los ritmos forrajeros de Atta laevigata F. Smith en una sabana, sus resultados confirmaron la hipótesis de que los ritmos de forrajeo en ambientes climáticamente estables están más relacionados con factores intrínsecos de cada colonia que por variables climáticas, las que poseerían un papel mas importante como limitante, y/ o disparador, que como regulador de la actividad de las hormigas. Sin embargo, no son escasos los trabajos en los que sí se ha visto un efecto significativo de algunos factores ambientales sobre el comportamiento de estos insectos. Algunos autores han observado una marcada relación entre la temperatura y la actividad de cosecha, generalemnte siguiendo variaciones térmicas diarias o estacionales (MINTZER, 1979; PILATI; QUIRÁN, 1996; ARAUJO et al., 2002; MELO et al., 2009). En el presente estudio, si bien las temperaturas máximas y mínimas no se relacionaron con la intensidad de forrajeo de A. lundi, no se podría afirmar que esta actividad es completamente independiente de la misma durante todo el año, debido a que las observaciones fueron realizadas sólo desde mitad de verano a principios de otoño. Por otra parte, Pilati y Quirán (1996) determinaron que la actividad diaria de $A$. lobicornis está íntimamente relacionada con la temperatura sólo en algunas épocas del año (otoño y primavera), mientras que en otras (verano), podría haber otros factores como nuevas fuentes de alimento afectando la actividad más que las condiciones ambientales.

Durante el período de observación, las temperaturas fueron en promedio para el primer año: ${ }^{\circ} \mathrm{T}$ máxima $25,5 \pm 1,5$ ${ }^{\circ} \mathrm{C} \mathrm{y}{ }^{\circ} \mathrm{T}$ mínima $13,3 \pm 1,1{ }^{\circ} \mathrm{C}$ y para el segundo año; ${ }^{\circ} \mathrm{T}$ máxima $25,3 \pm 0,6{ }^{\circ} \mathrm{C} \mathrm{y}{ }^{\circ} \mathrm{T}$ mínima $14,1 \pm 0,6{ }^{\circ} \mathrm{C}$. Este rango se corresponde con el que menciona Link (1997) como el de mayor actividad para Acromyrmex, lo que coincide con el contínuo forrajeo observado durante ambos períodos de estudio.

La cosecha de hojas aumentó cuando hubo mayor número de días con precipitaciones entre una observación y la siguiente. El coeficiente de determinación indica que casi el $40 \%$ de la variabilidad del número de hojas cosechadas podría ser explicado a través de esta variable. Si bien Wirth et al. (1997) observaron una correlación negativa entre la cosecha diaria y la duración de la lluvia, aunque estos mismos autores observaron también que máxima actividad de forrajeo se produce previamente a la ocurrencia de las precipitaciones. Por su parte, Sanchez Galarza y Urcuqui Bustamante (2006) observaron que la humedad parece tener una influencia positiva sobre la intensidad de cosecha en las colonias de Atta cephalotes (L.), ya que las distancias de forrajeo son mayores en los meses de lluvia, lo que fue atribuído al estrés fisiológico y menor disponibilidad del recurso durante los meses de sequía. Similares resultados fueron observados también en otras especies (WERNER, 1973; ARAUJO et al., 2002).

La herbivoría afectó el crecimiento en altura, el aumento del área foliar y el diámetro final de todas las procedencias. Algunos autores observaron similares resultados en esta especie de eucalipto, cuando ejercieron niveles de defoliación de 25 y $38 \%$ (PINKARD et al., 2007). Sin embargo, Collett y Neumann (2002) efectuaron artificialmente distintos niveles de defoliación, encontrando que sólo el 100\% redujo significativamente la altura y el diámetro basal del tallo de plantas de E. globulus durante la implantación, mientras que la defolición parcial no influyó sobre el crecimiento e incluso la remoción de las hojas inferiores favoreció el aumento de altura.En este trabajo la defoliación observada nunca alcanzó el 100\% de las hojas (datos no presentados) y, sin embargo, las plantas con herbivoría redujeron, con respecto a las plantas control, en promedio un $10,3 \%$ y un $26,5 \%$ el diámetro final y el crecimiento en altura, respectivamente. Esto podría explicarse a través de las observaciones realizadas por Quentin et al. (2010), quienes encontraron que la defoliación producidapor insectos en plantines de E. globulusde similar edad que los utilizados en este ensayo, redujo significativamente su altura y diámetro, mientras que la artificial sólo afectó el diámetro, concluyendo que las defoliaciones simuladas podrían subestimar el efecto de la herbivoría.

Es importante destacar que la altura y el diámetro son dos de las principales medidas morfológicas de la calidad de un árbol en vivero, entendiéndose por "calidad de una planta forestal" a la capacidad de la misma para alcanzar suficientes expectativas de supervivencia y de crecimiento posterior en el monte (SERRADA HIERRO, 2000). Aparicio et al. (2002) comprobaron que la altura inicial de los plantinesde E. grandis explica el $66 \%$ de la variación del crecimiento a los 24 meses de edad de los árboles. Por tanto, el

Revista Árvore, Viçosa-MG, v.39, n.1, p.189-198, 2015

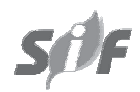


efecto de la defoliación sobre el crecimiento durante los años de implantación de un monte podría repercutir en su producción a largo plazo. En este sentido, Zanetti et al. (2000) encontraron una relación directa entre la densidad de nidos de hormigas cortadoras y la reducción de la producción de madera de Eucalyptus en Brasil durante cinco años.

Si bien era esperable que las plantas con herbivoría tuvieran un menor aumento del área foliar por ser la defoliación una causa directa de su reducción, ambas variables, número de hojas cosechadas y reducción del área foliar, no se correlacionaron significativamente $(\mathrm{R}=0,14, \mathrm{p}=0,67)$. Dos ejemplos ilustran claramente esta situación. Por un lado, la procedencia Bolaro fue la que presentó mayor reducción del área foliar, además de altura y diámetro, y sin embargo no fue una de las más cochadas por las hormigas. En el otro extremo, Jeeralang estuvo entre las procedencias más cosechas durante los dos años y al mismo tiempo fue una de las tres con menor reducción del área foliar (Tabla 2, Figura 2). Esto podría indicar que el efecto de la herbivoría sobre el crecimiento de las plantas depende más de la capacidad de cada procedencia de compensar la defoliación que del nivel de cosecha sufrido. El distinto comportamiento de las plantas frente a la herbivoría podría ser una característica asociada al genotipo de cada procedencia. Se ha observado que las plantas de E. globulus sometidas a defoliación natural y artificial, aumentan la capacidad fotosintética del follaje remanente y modifican la partición de los asimilados (PIKARD et al., 2007; QUENTIN et al., 2010). Como en este trabajo todos los brotes axilares fueron removidos en cada observación, el principal órgano destino de los fotoasimilados fue el brote apical, cuyo desarrollo favorece el crecimiento en altura y la emisión de nuevas hojas, aumentando el área foliar. Por lo tanto, favorecer la partición de asimilados hacia el desarrollo del brote apical y aumentar la eficiencia fotosintética del follaje remanente, podrían haber sido las estrategias de los genotipos que compensaron en mayor medida la herbivoría.

Ferrel y Floyd (2007) mencionan que los mecanismos de resistencia genética de las plantas se suelen agrupar en tres categorías: no preferencia, antibiosis y tolerancia. La no preferencia se produce cuando los insectos tienden a evitar o reducir su tasa de alimentación de una especie o genotipo; la antibiosis ocurre cuando existen barreras físicas y, o, químicas que afectan el desempeño o limitan el consumo del vegetal y por último la tolerancia se presenta en algunas plantas que siguen creciendo bien y con rendimientos elevados incluso cuando mantienen un nivel de daño que reduciría los rendimientos en otros genotipos. El primero de estos fenómenos no se observó en este trabajo, debido a que las hormigas forrajearon sobre todos los materiales genéticos durante los dos años. En cuanto a la antibiosis, A. lundi sólo presentó selectividad entre las procedencias E. globulus globulus Flinders Island y E. globulus bicostata Nullo Mountain, lo que sugiere la presencia de alguna barrera física y/o química en este último material menos elegido.

Por último, la tolerancia de las procedencias parece ser un factor muy importante en la resistencia de los materiales genéticos. Analizando en su conjunto las Figuras 1 y 2 , surge que no hay una evidente relación entre la mayor herbivoría y la reducción del crecimiento, lo que sugiere un diferente grado de compensación a la defoliación dado por el genotipo de cada procedencia.

\section{CONCLUSIÓN}

Los resultados obtenidos permiten concluir que la actividad de Acromyrmex lundi en relación a las procedencias de Eucalyptus globulus evaluadas es escasamente selectiva, que el crecimiento de las plantas es afectado independientemente del material genético y que la respuesta de compensación de las plantas a la herbivoría debería ser el principal factor a tener en cuenta a la hora de seleccionar materiales genéticos para una determinada región. En este sentido, procedencias como Bolaro y Murray de E. globulus maidenii y Wee Jasper de E. globulus bicostata, resultaron muy poco tolerantes a la herbivoría, lo que se tradujo en una marcada reducción del crecimiento, por lo que no serían recomendables en sitios con abundancia de ésta u otras especies defoliadoras.

Futuros estudios deberían explorar las características físicas y químicas de la procedencia Nullo Mountain de la subespecie E. globulus bicostata, para determinar las posibles causas de la menor herbivoría registrada en dicho material.

\section{REFERENCIAS}

ALVES, M. A. R.; DELlA LUCIA, T. M. C. Is Eucalyptus citriodora attacked by leaf-cutting ants?. Revista Árvore, v.23, n.1, p.69-74, 1999.

Revista Árvore, Viçosa-MG, v.39, n.1, p.189-198, 2015 
APARICIO, J.; DOMEQ, J.; STAFFIERI, G.; PARISI, L. Relación entre el tamaño inicial de las plantas y el rendimiento de Grevillea robusta, Pinus taeda y Eucalyptus globulus. In: JORNADAS FORESTALES DE ENTRE RÍOS, 17. 2002, Concordia. Procedings... Disponível em: http://64.76.123.202/new/0-0/forestacion/_archivos/ biblioteca/

174\%20\%20Aparicio\%20trab\%20compl.pdf

ARAUJO, M. S.; DELlA LUCIA, T. C.; LIMA, C. A.; SOUZA, D. J.; PETTERNELLI, E. F. Foraging activity of Acromyrmex laticeps nigrosetosus Forel (Hymenoptera: Formicidae) in Eucalyptus Stand. Acta Scientiarum, v.24, n.5, p.13211325, 2002.

BARÓN LÓPEZ, F. J.; TÉLLEZ MONTIEL, F. Regresión múltiple. In: BARÓN LÓPEZ, F. J.; TÉLlEZ MONTIEL, F. (Ed.). Apuntes de bioestadística: Tercer ciclo de en ciencias de la Salud y Medicina. México: Universidad de Málaga, 2004. p.35-43. Disponível em: http:// www.bioestadistica.uma.es/baron/apuntes/ ficheros/cap06.pdf

CAFFARINI, P.; PELICANO, A.; CARRIZO, P.; LEMCOFF, J. H. Impacto del estrés hídrico y la procedencia de Eucalyptus globulus Labill. sobre el comportamiento de herbivoría de Acromyrmex lundi Guérin. IDESIA, v.24, n. 1, p. 7-11, 2006.

COLLETT, N. G.; NEUMANN, F. G. Effects of simulated chronic defoliation in summer on growth and survival of blue gum (Eucalyptus globulus Labill) within Young plantations in northern Victoria. Australian Forestry, v.65, n.2, p.99-106, 2002.

DELABIE, J. H. C.; OSPINA, M.; ZABALA, G. Relaciones entre hormigas y plantas: una introducción. In: FERNÁNDEZ, F. (Ed.).

Introducción a las hormigas de la región Neotropical. Bogotá: Instituto de Investigaciones de Recursos Biológicos Alexander Von Humbolt, 2003. p.167-180.

DELLA LUCIA, T. C. M. Hormigas de importancia económica de la Región Neotropical. In: FERNÁNDEZ, F. (Ed.). Introducción a las hormigas de la Región Neotropical. Bogotá: Instituto de Investigaciones de Recursos Biológicos Alexander Von Humbolt, 2003. p.337-349.
DIMARCO, R.; RUSO, G.; FARJI-BRENER, A. G. Patrones de herbovoría en seis especies leñosas del bosque templado de América del Sur: evidencia preliminar a favor de la hipótesis del balance carbono-nitrógeno. Ecología Austral, v.14, n.1, p.39-43, 2004.

FARJI BRENER, A. G. Influencia de la estacionalidad sobre los ritmos forrajeros de Atta laevigata (Hymenoptera: Formicidae) en una sabana tropical. Revista de Biología Tropical, v.41, n.3, p.897-899, 1993.

FERREL, R.; FLOYD, G. Impact of insects on Eucalyptus plantations in the Murray Valley. RIRDC/L\&WA/FWPRDC/MDBC, Joint Venture Agroforestry Program, 2007.

FLOYD, G.; FARROW, R. A.; MATSUKI, M. Within species variation in insect damage and grotw in Eucalyptus globulus. In: FERREL, R.; FLOYD, G. (Ed.). Impact of insects on Eucalyptus plantations in the Murray Valley. RIRDC/L\&WA/FWPRDC/MDBC, Joint Venture Agroforestry Program, 2007. p.44-55.

FRANZEL, C.; FARJI BRENER, A. ¿Oportunistas o selectivas? Plasticidad en la dieta de la hormiga cortadora Acromyrmex lobicornis en el noroeste de la Patagonia. Ecología Austral, v.10, n.1, p.159-168, 2000.

FOLGARAIT, P. J.; FARJI BRENER, A. G.; PROTOMASTRO, J. J. Influence of biotic, chemical and mechanical plant defenses on the foraging pattern of the leaf-cutter ant (Acromyrmex striatus) in a subtropical forest. Ecología Austral, v.4, n.1, p.11-17, 1994.

GOMIDES, C. H.; DELLA LUCIA, T. M.; ARAUJO, F. S.; MOREIRA, D. D. Velocidad de forrajeo y area foliar transportada por la hormiga Acromyrmex subterraneus (Hymenoptera: Formicidae). Revista de Biología Tropical, v.45, n.4, p.1663-1667, 1997.

GUARNASCHELLI, A. B. Efecto del ajuste osmótico y la elasticidad de la pared celular sobre el crecimiento de Eucalyptus globulus en relación con su establecimiento. 2009. Tesis (Maestría en Recursos Naturales) - Facultad de Agronomía, Universidad de Buenos Aires, Ciudad Autónoma de Buenos Aires, 2009. 
HOWARD, J. J. Leafcutting and diet selection: the role of nutrients, water, and secondary chemistry. Ecology, v.68, p.503-515, 1987.

LINK, D. Hormigas cortadoras, hábitos y su control. In: JORNADAS FORESTALES DE ENTRE RÍOS, 12., 1997, Concordia, 1997. Procedings... Disponível em: http:// 64.76.123.202/new/0-0/forestacion/_archivos/ _biblioteca/68\%20I\%201ink\%2097.pdf

MARSARO JÚNIOR, A. L.; MOLINARUGAMA, A. J.; LIMA, C. A.; DELLA LUCIA, T. M. Preferência de corte de Eucalyptus spp. por Acromyrmex laticeps nigrosetosus Forel, 1908 (Hymenoptera, Formicidae) em condiçöes de laboratorio. Ciência Florestal, v.17, n.2, p.171-174, 2007.

MELO, P. J. C.; RIBEIRO, G. T.; BARRETTO GONCALVEZ, G.; MENDONCA, P. D.; POLANCZYK, R. A.; ZANETTI, R.; SERRANO, J. E.; ZANUNCIO, J. C. Nest and foraging characteristics of Acromyrmex landolti balzani (Hymenoptera: Formicidae) in Northeast Brazil. Sociobiology, v.54, n.2, p.361-371, 2009.

MINTZER, A. Foraging activity for the Mexican leafcutting ant Atta mexicana (F. Smith), in a Sonoran Desert habitat (Hymenoptera, Formicidae). Insectes Sociaux Pari, v.26, n.4, p.364-372, 1979.

NICHOLS-ORIANS, C. M. The effects of light on foliar chemistry, growth and susceptibility of seedlings of a canopy tree to an attine ant. Oecologia, v.86, p.552-560, $1991 \mathrm{a}$.

NICHOLS-ORIANS, C. M. Enviromentally induced differences in plant traits: consequences for susceptibility to a leafcutter ant. Ecology, v.72, p.1609-1623, 1991 b.

OHMART, C. P.; EDWARDS, P. B. Insect herbivory on Eucalyptus. Annual Review of Entomology, v.36, p.637-657, 1991.

PELOTTO, J. P.; DEL PERO DE MARTÍNEZ, M. A. Chemical defenses in the tree Ziziphus mistol against the leaf-cutting ant Acromyrmex striatus. Ecología Austral, v.12, n.1, p.11-18, 2002.
PILATI, A.; QUIRÁN, E. M. Patrones de cosecha de Acromyrmex lobicornis (Formicidae: Attini) en un pastizal del Parque Nacional Lihué Calel, La Pampa, Argentina. Ecología Austral, v.6, n.1, p.123-126, 2002.

PILATI, A.; QUIRÁN, E. M.; ESTELRICH, H. D. Actividad forrajera de Acromyrmex lobicornis Emery (Hymenoptera: Formicidae) en un pastizal natural semiárido de la provincia de La Pampa (Argentina). Ecología Austral, v.7, n.1, p.49-56, 1997.

PINKARD, E. A.; BATTAGLIA, M.; MOHAMED, C. L. Defoliation and nitrogen effects on photosynthesis and growth of Eucalyptus globulus. Tree Physiology, v.27, p.10531063, 2007.

QUENTIN, G. Q.; PINKARD, E. A.; BEADLE, C. L.; WAERDLAW, T. J.; O'GRADY, A. P.; PATERSON, S.; MOHAMED, C. L. Do artificial and natural defoliation have similar effects on physiology og Eucalyptus globulus Labill. Seedlings? Annals of Forest Science, n.67, 2010. Disponível em: www.afs-journal.org DOI: $10.1051 /$ forest $/ 2009096$

ROCKWOOD, L. L. Plant selection and foraging patterns in two species of leaf-cutting ants (Atta). Ecology, v.57, n.1, p.48-61, 1976.

RODRIGUEZ J.; CALLED, Z.; MONTOYALERMA, J. Herbivoría de Atta cephalotes (Hymenoptera: Myrmicinae) sobre tres sustratos vegetales. Revista Colombiana de Entomología, v.34, n.2, p.156-162, 2008.

SAGPyA. Inventario nacional de plantaciones forestales en macizo. Buenos Aires, Argentina: Secretaría de Agricultura, Pesca y Alimentación, 2001.64p.

SÁNCHEZ GALARZA, J. A.; URCUQUI BUSTAMANTE, A. M. Distancia de forrajeo de Atta cephalotes (L.) (Hymenoptera, Formicidae) en el Bosque seco tropical del Jardín Botánico de Cali. Boletín del Museo de Entomología de la Universidad del Valle, v.7, n.1, p.1-9, 2006.

SANTANA, D. L. Q.; COUTO, L. Resistencia intra-específica a formigas cortadeiras.

Revista Árvore, Viçosa-MG, v.39, n.1, p.189-198, 2015 
Boletim de Pesquisa Florestal, v.20, p.13-21, 1990.

SERRADA HIERRO, R. Generalidades sobre viveros forestales. In: Apuntes de repoblaciones forestales. Madrid: FUCOVASA, 2000. Disponível em: http:// www.secforestales.org/web/images/serrada/ v1 textoviveros.pdf

SKOLMEN, R. G.; LEDING, F. T. Eucalyptus globulus Labill. Bluegum eucalyptus In: BURNS, R. M.; HONKALA, B. H. (Ed.). Silvics of North America: 2. Hardwoods. Washington: U.S., Department of Agriculture, Forest Service, 1990. p.299-304.

SOUZA-SILVA, A.; ZANETTI, R. Forrageamento por Atta sexdens rubropilosa Forel, 1908

(Hymenoptera: Formicidae) a campo em mudas de eucalipto pulverizadas ou imersas em soluções de extrato pirolenhoso. Revista Árvore, v.31, n.4, p.753-759, 2007.

STATSOFT. Statistica for Windows.

Computer program manual. Tulsa: StatSoft, 2000.

STEEL, R. G. D.; TORRIE, J. H. Analysis of variance IV: Split-Plot designs and analysis. In:
STEEL, R. G. D.; TORRIE, J. H. (Ed.). Principles and procedures of statistics. A Biometricla approach. Singapore: Mc graw-Hill, 1980. p.377398 ,

VILLEGAS, M. S.; RIVERA, S. M. Revisión xililógica de las principals especies del género Eucalytus L'Herit. cultivadas en Argentina. Revista de la Facultad de Agronomía, La Plata, v.105, n.1, p.9-28, 2002.

WERNER, F. G. Foraging activity of the leaf-cutting ant Acromyrmex versicolor, in relation to season, weather and colony conditions. Logan: US International Biology Program, Desert Biome, 1973. p.73-28.

WIRTH, R.; BEYSCHLAG, W.; RYEL, R. J.; HOLLDOBLER, B. Annual foraging of the leafcutting ant Atta colombica in a semideciduous rain forest in Panama. Journal of Tropical Ecology, v.13, p.741-757, 1997.

ZANETTI, R.; JAFFÉ, K.; VILELA, E. F.; ZANUNCIO, J. C.; LEITE, H. G. Efeito da densidade e do tamanho de sauveiros sobre a produção de madeira em eucaliptais. Anais da Sociedade Entomógica do Brasil, v.29, n.1, p.105-112, 2000. 